\title{
Brain natriuretic peptide could be a biomarker for outcomes after cardioembolic stroke
}

Serum levels of brain natriuretic peptide (BNP) show a significant correlation with mortality and long-term functional outcomes after cardioembolic stroke, according to a large, prospective cohort study. "Given the complex interactions between multiple factors, predicting longterm functional outcomes after stroke remains one of the most challenging issues in the field," says Natalia Rost, who was involved in the study.

$\mathrm{BNP}$ is released by the heart in response to excessive stretching of cardiomyocytes, and is validated as a biomarker for outcomes in cardiovascular diseases such as heart failure. Prior to the current study, the predictive utility of this peptide in the context of stroke had been unclear.

To address this issue, Rost and colleagues measured the serum BNP levels at hospital admission of consecutive patients with ischemic stroke. Transthoracic echocardiography was also performed to measure cardiac parameters such as left ventricular ejection fraction and left atrium diameter. A stroke neurologist, blinded to the BNP data, assigned each patient a stroke subtype according to Trial of ORG 10172 in Acute Stroke Treatment (TOAST) criteria. After 3-6 months, telephone interviews were conducted to assess functional outcomes using the modified Rankin Scale.

"Elevated BNP levels were associated with significantly decreased odds of good functional outcome-for example, functional independence after stroke," says Rost. The study is the first to show this correlation. Raised serum BNP levels were also associated with a lower ejection fraction and with left atrial dilatation, and were an independent predictor of mortality.

When the data were analyzed according to stroke subtype, the association of BNP level with outcome and mortality was present only in cardioembolic stroke, and did not show a significant correlation in non-cardioembolic subtypes.

\author{
4 Elevated BNP levels were \\ associated with significantly \\ decreased odds of good \\ functional outcome... 77
}

Rost and colleagues also found that incorporation of BNP levels into existing models for prediction of outcome in cardioembolic stroke significantly increased the accuracy of predictions. Such improvements could lead to moreeffective stratification and treatment of patients with cardioembolic stroke. "Further studies are required to validate the findings in an independent cohort," concludes Rost.

Katie Kingwell

Original article Rost, N. S. et al. Brain natriuretic peptide predicts functional outcome in ischemic stroke. Stroke doi:10.1161/STROKEAHA.111.629212 